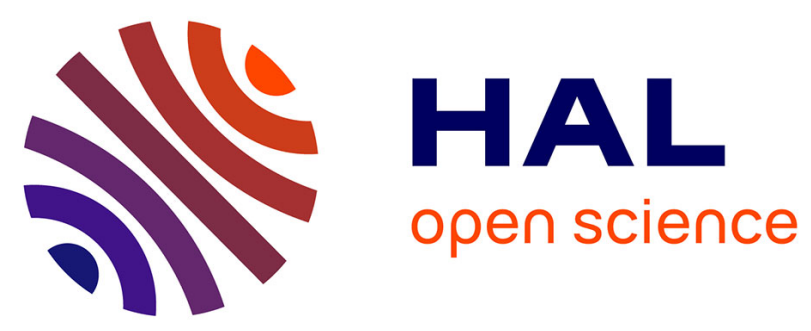

\title{
Investigation of the effect of double-walled carbon nanotubes on the curing reaction kinetics and shear flow of an epoxy resin
}

Ihab El Sawi, Philippe Olivier, Philippe Demont, Habiba Bougherara

\section{- To cite this version:}

Ihab El Sawi, Philippe Olivier, Philippe Demont, Habiba Bougherara. Investigation of the effect of double-walled carbon nanotubes on the curing reaction kinetics and shear flow of an epoxy resin. Journal of Applied Polymer Science, 2012, vol. 126, pp.358-366. 10.1002/app.36988 . hal-00825640

\author{
HAL Id: hal-00825640 \\ https://hal.science/hal-00825640
}

Submitted on 24 May 2013

HAL is a multi-disciplinary open access archive for the deposit and dissemination of scientific research documents, whether they are published or not. The documents may come from teaching and research institutions in France or abroad, or from public or private research centers.
L'archive ouverte pluridisciplinaire HAL, est destinée au dépôt et à la diffusion de documents scientifiques de niveau recherche, publiés ou non, émanant des établissements d'enseignement et de recherche français ou étrangers, des laboratoires publics ou privés. 


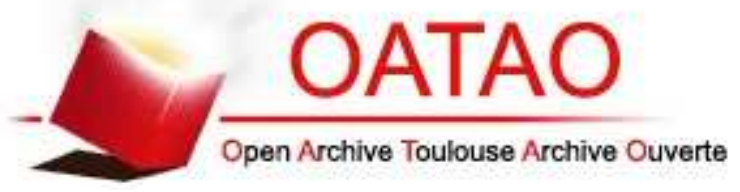

\section{Open Archive TOULOUSE Archive Ouverte (OATAO)}

OATAO is an open access repository that collects the work of Toulouse researchers and makes it freely available over the web where possible.

This is an author-deposited version published in : http://oatao.univ-toulouse.fr/ Eprints ID : 8708

To link to this article : DOI:10.1002/app.36988

URL : http://dx.doi.org/10.1002/app.36988

To cite this version :

El Sawi, Ihab and Olivier, Philippe A. and Demont, Philippe and Bougherara, Habiba Investigation of the effect of double-walled carbon nanotubes on the curing reaction kinetics and shear flow of an epoxy resin. (2012) Journal of Applied Polymer Science, vol. 126 (n¹). pp. 358-366. ISSN 0021-8995

Any correspondence concerning this service should be sent to the repository administrator: staff-oatao@ listes.diff.inp-toulouse.fr 


\title{
Investigation of the Effect of Double-Walled Carbon Nanotubes on the Curing Reaction Kinetics and Shear Flow of an Epoxy Resin
}

\author{
Ihab El Sawi, ${ }^{1,2}$ Philippe A. Olivier, ${ }^{2}$ Philippe Demont, ${ }^{3}$ Habiba Bougherara ${ }^{1}$ \\ ${ }^{1}$ Department of Mechanical and Industrial Engineering, Ryerson University, Toronto, Ontario, Canada M5B 2K3 \\ ${ }^{2}$ Institut Clément Ader, Université de Toulouse, 133 C Avenue de Rangueil, 31077 Toulouse, Cedex 4, France \\ ${ }^{3}$ Institut Carnot CIRIMAT, Université de Toulouse, 118 route de Narbonne, 31062 Toulouse Cedex 9, France
}

\begin{abstract}
In this article, the effect of combined temperature-concentration and shear rate conditions on the rheology of double-walled carbon nanotubes (DWCNTs)/ RTM6-Epoxy suspension was investigated to determine the optimum processing conditions. The rheological behavior and cure kinetics of this nanocomposite are presented. Cure kinetics analysis of the epoxy resin and the epoxy resin filled with DWCNTs was performed using Differential Scanning Calorimeter (DSC) and parameters of the kinetics model were compared. The DWCNTs have an acceleration effect on the reaction rate of the epoxy resin but no significant effect is noted on the glass transition
\end{abstract}

temperature of the epoxy resin. This study reveals that the effect of shear-thinning is more pronounced at high temperatures when DWCNTs content is increased. In addition, the steady shear flow exhibits a thermally activated property above $60^{\circ} \mathrm{C}$ whereas the polymer fluid viscosity is influenced by the free volume and cooperative effects when the temperature is below $60^{\circ} \mathrm{C}$.

Key words: differential scanning calorimetry; shear viscosity; cure kinetics; carbon nanotubes/epoxy; glass transition

\section{INTRODUCTION}

Carbon nanotubes (CNTs) and CNTs-based composites are new emerging materials for structural and electrically conductive applications. ${ }^{1}$ The main advantage of CNTs is that the use of low percent of CNTs in polymer can enhance the strength, improve the stiffness, the thermal stability, and electrical conductivity of the polymer. ${ }^{2,3}$ Manufacturing such composites involves a good knowledge of processing parameters such as reaction kinetics and rheological behavior of the polymer-composite and the dispersion state of CNTs to define appropriate parameters for manufacturing the Polymer/nanotubes composites. Aiming for processing a structure made by carbon fibers and epoxy resin filled with carbon nanotubes the understanding of the changing of matrix behavior, from its monomer state (uncured resin) till the final cured matrix, is an important issue.

Correspondence to: I. El Sawi (ihab.elsawi@ryerson.ca or ihab.elsawi@gmail.com).

Contract grant sponsors: Scientific Council of Paul Sabatier University-Toulouse, The Ministry of Research and Innovation (ERA-program), Ontario.
Various techniques of processing CNTs/epoxy composites have been presented in the literature. ${ }^{4}$ However, to make CNTs properties more advantageous, their dispersion in the polymer matrix must be controlled. Although chemically treated CNTs (such as amino-functionalized CNTs) could be easily dispersed and displayed good interfacial interactions with the polymer matrix, ${ }^{5-7}$ such treatment is detrimental to the electrical conductivity level. ${ }^{8,9}$ By contrast, Barrau et al. ${ }^{10}$ showed that a dispersion process involving an amphiphilic molecules (palmitic acid) allowed to achieve a good dispersion and then a lower electrical percolation threshold (i.e., $0.05 \mathrm{wt}$ $\%$ of CNTs) and a higher electrical conductivity of nanocomposite.

During its cure, the epoxy monomers react with an appropriate curing agent (generally containing amine groups) to form a three-dimensional crosslinked network with a certain thermomechanical properties. The degree and uniformity of curing reaction will affect considerably the bulk material properties. Regarding the cure kinetics, a large number of published papers dealt with the effect of CNTs and functionalized CNTs on the cure kinetics and the glass transition temperature of the epoxy resin. ${ }^{11-18}$ Zhou et al. ${ }^{11}$ showed that both unfunctionalized and functionalized multiwalled carbon nanotubes (MWCNTs) have an accelerating influence on 
the reaction kinetics. They also found that the degree of cure is decreased by the addition of unfunctionlized MWCNTs. This result was evidenced by the lower value of the $T_{g}$ of the cured nanocomposite compared with the neat epoxy. For example 1\% of unfunctionlized MWCNTs reduces the $T_{g}$ of the neat epoxy by $15^{\circ} \mathrm{C}$.

Xie et al. ${ }^{12}$ studied the effect of MWCNTs on cure kinetics of the tetraglycidyl ether of a diaminodiphenylmethane/diaminodiphenylsulfone (TGDDM/DDS) epoxy/amine system using isothermal DSC. Their results showed that MWCNTs increase the initial reaction rate of the resin, decrease the time at maximum reaction rate and decrease the activation energy. These effects were proportional to MWCNTs content in the epoxy resin which indicates an acceleration effect of the MWCNTs. This catalytic effect was assigned to the hydroxyl $(-\mathrm{OH})$ groups on the surface of the MWCNTs. On the other hand, Puglia et al. ${ }^{13}$ showed that the incorporation of single-walled carbon nanotubes (SWCNTs) affects the cure reaction of a diglycidyl ether of bisphenol A epoxy matrix. It was observed that the rate of reaction increased with increasing SWCNTs concentration. They Interpreted this effects in terms of the extremely high-thermal conductivity of carbon nanotubes which assist the heat propagation. The study of Tao et al. ${ }^{14}$ about the effect of SWCNTs on the diglycidyl ether of bisphenol F/diethyltoluenediamine (DGEBF/DETD) epoxy/amine system revealed a decrease of the onset temperature in dynamic scans and a decrease of the time at maximum reaction in isothermal scans. The measured $T_{g}$ of their samples showed that the $T_{g}$ of the composites was always lower than the unfilled epoxy. They attributed this to the lower curing degree of the composites.

Furthermore, if a bulk processing of nanotubepolymer suspension is to be carried out, a well understanding of the responses of the suspension to simple steady-state shear flow is required. Regarding the rheological behavior of CNTs filled epoxy resin many papers have dealt with the effect of the microstructure, dispersion state, and orientation of CNTs on the rheology of suspension of CNTs in polymers. ${ }^{19,20}$ Other studies dealt the effect of CNTs content and their chemical modification on the shear viscosity of the polymer. ${ }^{2,21}$ CNTs induce shear thinning of the Polymer matrix. An increase of the CNTs content leads to an increase of the shear thinning behaviors. Nanocomposites containing modified CNTs exhibit higher shear viscosity than those with the untreated CNTs, because surface treatments induce more homogeneous dispersion of CNTs and stronger interaction between CNTs and the polymer matrix. ${ }^{2}$ More recent papers investigated the effect of shear flow on the physical properties and microstructure of CNTs/polymer suspensions ${ }^{22,23}$ and particularly on the electrical conductivity. ${ }^{24-26}$
Aiming to point-out how might double-walled carbon nanotubes (DWCNTs) influence the reaction kinetics and the flow properties of a commercial epoxy resin; in this paper we tackle the effect of processing conditions on the performance of DWCNTs by studying their cure kinetics and rheological behavior. Differential scanning calorimetry and shear flow experiments were performed to determine the curing kinetic changes, cure parameters and resin viscosities induced by DWCNTs. The RTM6 epoxy resin is a high-grade epoxy used to manufacture high-performance epoxy-based composites for the aerospace industry. The properties and cure kinetics of this resin has been wildly studied. ${ }^{27-30}$ This is the first study to characterize the effect of carbon nanotubes on the cure kinetics, glass transition temperature $T_{g}$ and shear flow behavior of this high $T_{g}$ epoxy resin. Regarding the rheology tests, we focus on the analysis and interpretation of rheological characteristics of different concentration of DWCNTs in the RTM6 epoxy resin submitted to different shear rates and temperature. To our understanding, no work has been yet undertaken to investigate the effect of combined temperatureconcentration and shear rate conditions on the rheology of CNTs/Epoxy suspension.

\section{MATERIALS AND EXPERIMENTAL DETAILS}

\section{Materials}

The carbon nanotubes used in this study are doublewalled (DWCNTs) from CIRIMAT. ${ }^{31}$ DWCNTs were synthesized by catalytic chemical vapor deposition (CCVD) with carbon content of $98 \mathrm{vol} \%$. According to statistical studies on HR-TEM images of CNTs, $18 \%$ are single-walled, $77 \%$ double-walled, and $5 \%$ triple-walled. The outer average diameter is $2 \mathrm{~nm}$ and a length $\sim 5 \mu \mathrm{m}$. The BET specific surface area of CNTs is $700 \mathrm{~m}^{2} \mathrm{~g}^{-1}$. 32 The aspect ratio (length/ diameter) of an individual carbon nanotube can be estimated at $5 \times 10^{3}$ to $1 \times 10^{4}$. After their extraction and purification, DWCNTs were washed many times with distilled water and kept in water prior dispersion in epoxy resin.

A monocomponent HexFlow RTM6 epoxy resin from Hexcel Composites was used as polymer matrix. According to the supplier, the density at $25^{\circ} \mathrm{C}$ of the uncured and cured epoxy resin is 1.11 and $1.14 \mathrm{~g} \mathrm{~cm}^{-3}$ respectively and the viscosity is 175 and $33 \mathrm{mPa}$ s at 25 and $80^{\circ} \mathrm{C}$ respectively. The dispersion of CNTs in the epoxy matrix is assisted by the use of hexadecyclamine [HDA: $\mathrm{CH}_{3}-(\mathrm{CH} 2)_{15} \mathrm{NH}_{2}$ ] from Sigma-Aldrich, as amphiphilic molecule. According to Barreau et al., ${ }^{10}$ the ration HDA: CNTs weight ratio was taken as 1: 1 . 


\section{Epoxy resin-DWCNTs suspension preparation}

For each weight fraction of DWCNTs in the composite we produced, HDA was added to an aqueous suspension of DWCNTs and then sonicated in ultrasonic bath for $1 \mathrm{~h}$ at room temperature and subsequently probe-tip sonicated for $15 \mathrm{~min}$ at $20 \mathrm{~W}$. The aqueous suspension DWCNTs/HDA was added to the epoxy resin and the mixture was stirred at a rotational speed of $1000 \mathrm{rpm}$ for $30 \mathrm{~min}$ at $80^{\circ} \mathrm{C}$. The mixture was degassed for $3 \mathrm{~h}$ at $80^{\circ} \mathrm{C}$ under vacuum to remove the solvent (water).

\section{Differential scanning calorimetry}

The curing kinetic study was performed using a TAInstruments DSC-Q100 differential scanning calorimeter. Isothermal and dynamic-heating experiments were conducted under a nitrogen flow. Cure kinetics study of the epoxy resin was performed by curing 6 mg samples under isothermal cure temperatures conditions, ranging from 100 to $180^{\circ} \mathrm{C}$ for times up to $14 \mathrm{~h}$. After isothermal curing, each sample was cooled to $-30^{\circ} \mathrm{C}$ at a rate of $20^{\circ} \mathrm{C} \mathrm{min}$ min $^{-1}$ and subsequently scanned by heating at heating rate of $10^{\circ} \mathrm{C}$ $\min ^{-1}$ up to $300^{\circ} \mathrm{C}$. From this scan, the glass transition temperature $T_{g}$ and the residual heat of reaction $\Delta H_{\text {res, }}$ of the partially cured samples were obtained. The total heat of reaction $\Delta H_{T}$ was obtained by scanning uncured samples from -30 to $300^{\circ} \mathrm{C}$ at a heating rate of $10^{\circ} \mathrm{C} \mathrm{min}^{-1}$. The glass transition temperature is taken to be the temperature of the midpoint of the step change in the heat capacity.

Prior conducting our experiments the heat flow and the temperature of the DSC have been calibrated using standard indium with known enthalpy of fusion and melting temperature. The calibration has been made over the range of studied temperature $\left(-30\right.$ to $\left.300^{\circ} \mathrm{C}\right)$ and at a temperature scanning rate of $10^{\circ} \mathrm{C} \mathrm{min}^{-1}$. The instrumental baseline was determined prior each experiment by scanning (comparing) two empty aluminium pans. Sealed aluminium sample pans were used for all our experiments. In order to minimize thermal gradients inside samples we used thin and low mass samples $\sim 0.7-\mathrm{mm}-$ thickness and $6 \mathrm{mg}$.

Modulated DSC (MDSC) permits the accurate measurement of heat capacity of a material when experimental conditions are optimized. In order to measure the evolution of the specific heat capacity $\left(C_{p}\right)$ of our samples during their curing cycle by MDSC, we calibrated the device using a standard sapphire provided by TA-Instruments with known heat capacity over the range of temperature that we are studying. We performed MDSC experiments on uncured neat RTM6 epoxy resin and uncured RTM6 epoxy resin filled with $0.4 \mathrm{wt} \%$ DWCNTs. The experiments were conducted in quasi-isothermal mode at $160^{\circ} \mathrm{C}$ using modulation periods of $100 \mathrm{~s}$. Aiming to obtain comparable results; our experiments wer performed on samples that have the same mass, that is, $6.4 \mathrm{mg}$.

\section{Rheology}

The shear flow characteristics of the epoxy resin suspensions with different amounts of DWCNTs were performed using a Carri-Med CSL2 500 Rheometer from TA-instruments. A 40-mm diameter parallelplate and 1-mm gap configuration was used. The steady shear viscosity was measured at a shear rate $\dot{\gamma}$ in the range of $0.2-120 \mathrm{~s}^{-1}$ and at temperature ranging from 25 to $80^{\circ} \mathrm{C}$.

\section{MODELS}

\section{Cure kinetics model}

The rate of conversion as function of time $d \alpha / d t$ and the fractional degree of conversion $\alpha(t)$ were determined using eqs. (1) and (2), respectively. $d \alpha / d t$ represents the main features of the reaction kinetics without taking into account the role of individual species separately. Therefore, $d \alpha / d t$ can represent the reaction rate of the epoxy system.

As a fundamental assumption, the recorded value of heat flow $d H / d t$ relative to the instrumental baseline is proportional to the reaction rate or rate of conversion:

$$
\frac{d \alpha}{d t}=\frac{d H}{d t} \frac{1}{\Delta H_{T}}
$$

and

$$
\alpha(t)=\frac{\int_{0}^{t} \frac{d H}{d t} d t}{\Delta H_{T}},
$$

where $H$ is enthalpy of the reaction at time $t$, and $\Delta H_{T}$ is the total heat of the cure reaction. A value of $\Delta H_{T}=430 \mathrm{~J} \mathrm{~g}^{-1}$ was determined from dynamic runs at a heating rate of $10^{\circ} \mathrm{C} \mathrm{min}^{-1}$.

Epoxy resins curing reactions can be described by $n$th order reaction kinetics and/or autocatalytic reaction kinetics. The reaction rate of the systems, obeying autocatalytic reaction, passes through a maximum and then decreases with increasing time as seen in Figure 1. In the cure kinetics model developed by Sourour et al., ${ }^{33}$ the conversion rate is a combination of an autocatalytic model and an $n$th order mode. ${ }^{34}$ We used a phenomenological model derived from Sourour and Kamal's model, developed by Lee et al. ${ }^{35}$ to study the cure kinetics of a 
high crosslinked epoxy/amine system namely TGDDM-DDS and expressed as follows:

$$
\frac{d \alpha}{d t}=k_{1}(T)\left(\alpha_{\max }-\alpha\right)^{n_{1}}+k_{2}(T) \alpha^{m}\left(\alpha_{\max }-\alpha\right)^{n_{2}},
$$

where $k_{1}$ and $n_{1}$ are the reaction rate constants and reaction order of the $n$th part respectively; $m$ and $n_{2}$ are the reaction orders of the autocatalytic part and $k_{2}$ the corresponding reaction rate constant. $\alpha_{\max }$ is the maximum degree of conversion at isothermal temperature.

The reaction rate constants $k_{i}$ are thermally activated and described by an Arrhenius equation:

$$
k_{i}(T)=A_{i} \exp \left(-\frac{E_{i}}{\mathrm{RT}}\right),
$$

where $E_{i}$ is an activation energy and $A_{i}$ is a pre-exponential factor.

Karkanas and Partridge ${ }^{29}$ and Kazilas et al. ${ }^{28}$ showed that this model Eq. (3) can describe suitably the RTM6 reaction kinetics. The cure reaction parameters were obtained from the non linear regression of the rate of conversion $d \alpha / d t$ versus $\alpha$ for each isothermal cure temperature using Eq. (3).

The fractional conversion $\alpha$ reaches an asymptotic value $\alpha_{\max }(T)$ due to the vitrification and diffusion controlled kinetics. Several expressions of $\alpha_{\max }(T)$ for epoxy systems have been proposed in the literature, for example empirical relations proposed by Torre et al. ${ }^{34}$ and Terenzi et al. ${ }^{36}$ Gonzalez-Romero ${ }^{37}$ proposed a modified form of the empirical equation developed by Di Benedetto ${ }^{38}$ to describe the temperature dependence of the maximum conversion $\alpha_{\max }$ in epoxy systems, see Eq. (5):

$$
\alpha_{\max }(T)=\frac{T_{g 0} T_{g \infty}}{T\left(T_{g 0}-T_{g \infty}\right)}+\frac{T_{g \infty}}{\left(T_{g \infty}-T_{g 0}\right)}
$$

In this equation, $T_{g} 0$ and $T_{g} \infty$ are the glass temperature of the uncured and completely cured epoxy resin and $T$ is the isothermal cure temperature.

\section{Rheological model}

To investigate the shear-thinning behavior of our CNTs/epoxy suspensions we used the CarreauYasuda ${ }^{39,40}$ model, see Eq. (6). Parameters of the model were studied in this section to describe the shear-thinning induced by DWCNTs.

$$
\frac{\eta-\eta_{\infty}}{\eta_{0}-\eta_{\infty}}=\left[1+(\tau \dot{\gamma})^{2}\right]^{\frac{n-1}{2}}
$$

where $\eta_{0}$ and $\eta_{\infty}$ refer to the asymptotic values of viscosity at very low and very high-shear rates, respectively, (or zero shear viscosity and infinite shear viscosity respectively), $n$ is the power law exponent (or pseudoplasticity index) characteristics of the deviation from the Newtonian behavior $(n=$ 1 ) and $\tau$ is a characteristic time of the shear-thinning behavior.

Thermally activated dependency of the $\eta_{0}$ appears for all materials at $T \geq 60^{\circ} \mathrm{C}$. The model to describe the evolution of $\eta_{0}$ with respect to temperature is the Andrade-Eyring ${ }^{41}$ model given by Eq. (7):

$$
\eta_{0}=\eta_{0}\left(T_{s}^{\prime}\right) \cdot \exp \left[\frac{\Delta E_{a}}{R} \cdot\left(\frac{1}{T}-\frac{1}{T_{s}^{\prime}}\right)\right]
$$

where $T_{s}^{\prime}$ represents the reference temperature and is taken to be $(60+273.15) \mathrm{K}, \eta_{0}\left(T_{s}^{\prime}\right)$ is the zeroshear viscosity at $T^{\prime}{ }_{s}$ estimated from experimental results. $\Delta E_{a}$ is the thermal activation energy for viscous flow and $R$ is the gas constant $8.314 \mathrm{~J} \mathrm{~mol}^{-1}$ $\mathrm{K}^{-1}$.

The shear flow property for $T<60^{\circ} \mathrm{C}$ is modeled with the Williams-Landel-Ferry ${ }^{42}$ (WLF) analytical model presented in Eq. (8) using $T_{S}=(28+273.15)$ $\mathrm{K}$ as reference temperature. The WLF equation is written as following:

$$
\begin{aligned}
\log \left(a_{T}\right)=\log \left(\frac{\eta_{0}(T)}{\eta_{0}\left(T_{S}\right)}\right) & =\left(\frac{-C_{1}^{S} \cdot\left(T-T_{S}\right)}{C_{2}^{S}+\left(T-T_{S}\right)}\right) \\
& \text { or } \frac{1}{\log \left(a_{T}\right)}=-\frac{1}{C_{1}^{S}}-\frac{C_{2}^{S}}{C_{1}^{S}} \cdot \frac{1}{\left(T-T_{S}\right)}
\end{aligned}
$$

The $\eta_{0}\left(T_{S}\right)$ is the zero viscosity at reference temperature $T_{S}$ and $a_{T}(T)$ is the time-temperature superposition shift factor. The WLF equation is derived on the basis of temperature-dependent free volume of Vogel-Fulcher-Tammann (VFT) ${ }^{43-45}$ in which the $C_{1}^{S}=B /\left(2.303 \times f_{s}\right)$ and $C_{2}^{S}=f_{s} / \alpha_{f}$. Where $f_{s}$ represents the fractional free volume at $T_{S}, \alpha_{f}$ is the thermal expansion coefficient of fractional free volume, and $B$ is a constant, generally assumed to be unity.

\section{RESULTS AND DISCUSSION}

\section{Cure kinetics}

To investigate the effect of DWCNTs on the cure kinetics of the RTM6 epoxy resin, DWCNTs weight fraction of 0.4 wt \% is taken as reference, because this value is higher than the electrical percolation threshold of the corresponding DWCNTs/epoxy composites and the viscosity of the filled resin at 0.4 wt \% of DWCNTs is still low to process DWCNTs/ epoxy composites. Figure 1 shows a series of exothermic reaction energy for neat epoxy resin and 0.4 wt \% DWCNTs filled epoxy resin from isothermal scans at $160,120,110$, and $100^{\circ} \mathrm{C}$. The fractional 


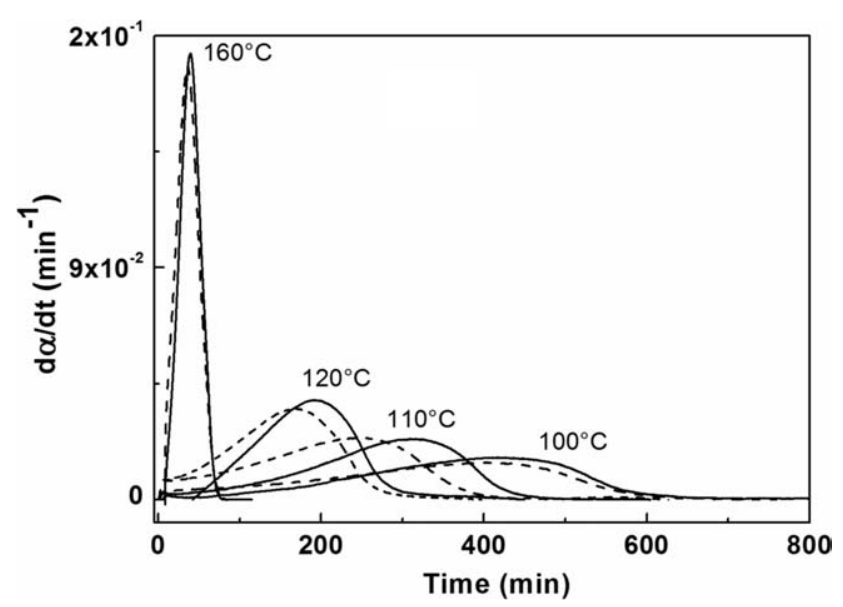

Figure 1 Rate of conversion $d \alpha / d t\left(\min ^{-1}\right)$ versus time (min) for neat RMT6 epoxy resin (-) and 0.4 wt \% DWCNTs filled RTM6 epoxy resin (---).

conversion $\alpha$ derived from Eq. (2) is represented as function of time in Figure 2.

The reaction rate at a nonzero time is observed for all the samples and temperatures. $d \alpha / d t$ versus time is characteristic of an autocatalytic behavior in the RTM6 epoxy systems. As expected the reaction rate passes through a maximum and then decreases as a function of curing time. The peak value of $d \alpha / d t(t)$ increases and shifts to shorter times with increasing cure temperature for all the samples. The onset of the reaction appears earlier for the resin filled with DWCNTs which are represented by dashed plot on Figure 1. DWCNTs dispersion in epoxy resin matrix induces a slight decrease of the maximum heat flow rate and the time to reach the maximum rate.

The fractional conversion $\alpha$ results presented in Figure 2 show the experimental degree of conversion as a function of the curing time. For an isothermal curing temperature and at given time, the fractional conversion is higher for the DWCNTs filled epoxy resin compared with the neat epoxy resin. This observation is true for low isothermal temperatures $T$ $<160^{\circ} \mathrm{C}$. For higher isothermal temperatures, the evolution of the fractional conversion versus time is similar for both materials. Although we believe that our purified DWCNTs are chemically inert under these thermal curing conditions, we expect that the thermal conductivity is increased by the presence of DWCNTs which can lead the reaction to begin earlier when the resin is filled with DWCNTs.

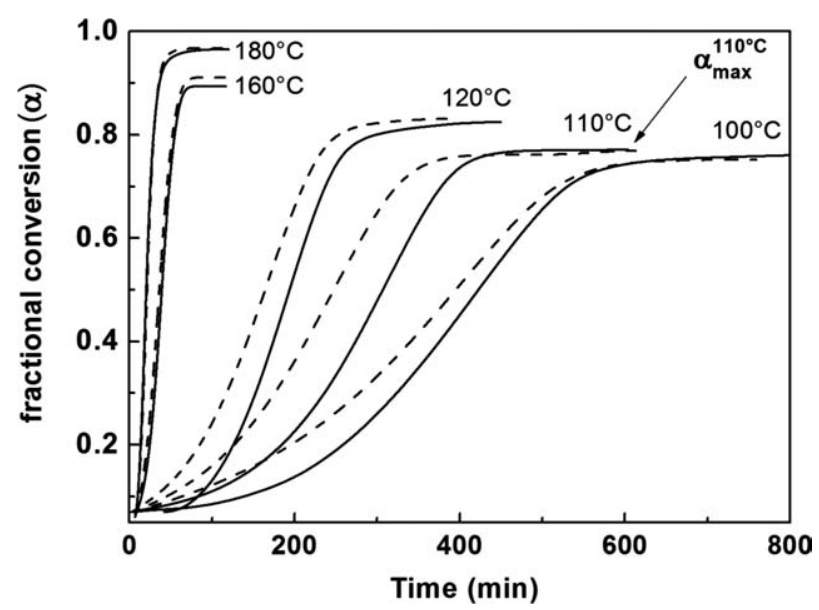

Figure 2 Fractional degree of conversion $\alpha$ as a function of curing time at various isothermal temperatures for neat RMT6 epoxy resin (-) and 0.4 wt \% DWCNTs filled RTM6 epoxy resin (---).

In order to study the parameters of the cure kinetics model of Eq. (3) we first determine the activation energies of the reaction, the constants $k_{1}$ and $k_{2}$ [Eq. (4)] and the reaction order $n_{1}, n_{2}$, and $\mathrm{m}$. The values of $A_{i}, E_{i}$, and $n_{1}$ are reported in Table I. The reaction order $n_{2}$ and $\mathrm{m}$ are found to be a linear function of isothermal temperature: $n_{2}=5.4 \times 10^{-3} \mathrm{~T}$ -0.94 and $\mathrm{m}=-2.1 \times 10^{-3} \mathrm{~T}+2.49$, $(\mathrm{T}$ in $\mathrm{K})$. Lee et $a .^{35}$ found a similar variation of the reaction order as function of temperature.

The activation energies for reaction are not affected by the presence of 0.4 wt \% DWCNTs whereas the time constants $A_{i}$ are significantly reduced $\left(-38.5 \%\right.$ for $\left.A_{1}\right)$ and $\left(-27.6 \%\right.$ for $\left.A_{2}\right)$.

The fractional conversion $\alpha(t)$ reaches an asymptotic value $\left(\alpha_{\max }\right)$ which depends only on the temperature. The maximum degree of conversion $\left(\alpha_{\max }\right)$ is plotted as function of the isothermal curing temperature in Figure 3 and fitted to the Eq. (5). The results show that the maximum degree of conversion is not affected by the presence of DWCNTs. By fitting the data in Figure 3 with Eq. (5) we determined $T_{g} 0=-33^{\circ} \mathrm{C} \pm 8^{\circ} \mathrm{C}$ and $T_{g} \infty=206^{\circ} \mathrm{C} \pm$ $7^{\circ} \mathrm{C}$. Theses values found theoretically by extrapolation can represent the real values of the glass transition temperature of the uncured $\left(\begin{array}{ll}T_{g} & 0\end{array}\right)$ and completely cured $\left(T_{g} \infty\right)$ epoxy resin.

In order to assess the effect of the DWCNTs on the thermal properties of the RTM6 epoxy resin, we

TABLE I

Cure Kinetics Parameters for Neat RTM6 Epoxy Resin and 0.4 wt \% DWCNTs/RTM6 Composite

\begin{tabular}{lcccrr}
\hline & $A_{1}\left(\mathrm{~s}^{-1}\right)$ & $A_{2}\left(\mathrm{~s}^{-1}\right)$ & $E_{1}\left(\mathrm{~kJ} \mathrm{~mol}^{-1}\right)$ & $E_{2}\left(\mathrm{~kJ} \mathrm{~mol}^{-1}\right)$ & $n_{1}$ \\
\hline RTM6 & $3.4 \times 10^{4}$ & $1.8 \times 10^{3}$ & $74 \pm 3$ & $47 \pm 3$ & 0.45 \\
DWCNTs/RTM6 & $2 \times 10^{4}$ & $1.3 \times 10^{3}$ & $72 \pm 3$ & $46 \pm 3$ & 0.45 \\
\hline
\end{tabular}




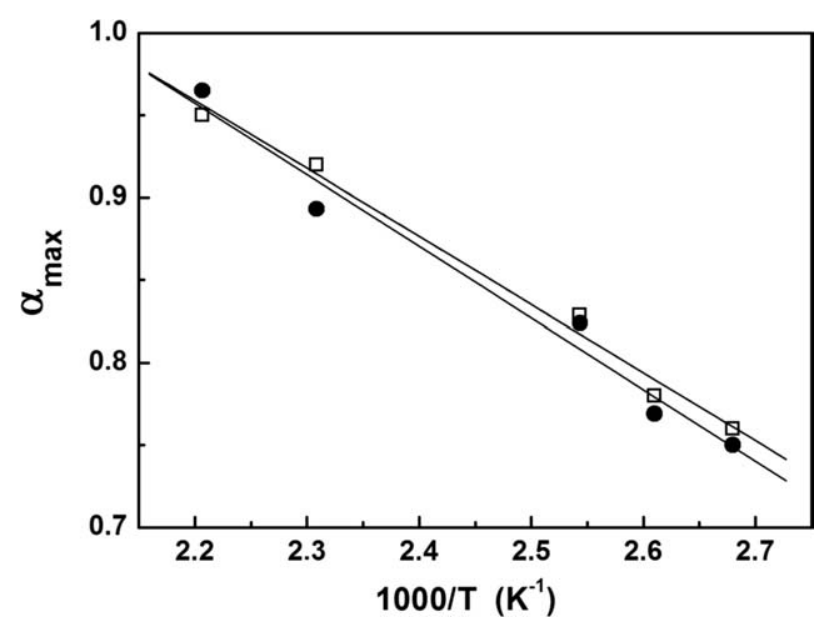

Figure 3 Maximum degree of conversion as function of $1000 / T ; T$ is the isothermal cure temperature. Neat RTM6 epoxy resin ( $\square), 0.4$ wt \% DWCNTs filled RTM6 epoxy resin mixture $(0)$ and the thin full lines represent the Di Benedetto model [Eq. (5)].

compare the heat capacities of the neat epoxy resin and the epoxy resin filled with 0.4 wt $\%$ of DWCNTs. Results are plotted in Figure 4. The onset of the vitrification appears at $63 \mathrm{~min}$ of the isothermal cure at $160^{\circ} \mathrm{C}$. The virtification appears at the same time for both materials. The mean values of $C_{p}$ are determined from the two pseudo-plateaux of the curves presented in Figure 4.

The heat capacities previous to vitrification are determined: $C_{p}=2.02 \mathrm{~J} \mathrm{~g}^{-1}{ }^{\circ} \mathrm{C}^{-1}$ for the RTM6 epoxy resin and $C_{p}=1.93 \mathrm{~J} \mathrm{~g} \mathrm{~g}^{-1}{ }^{\circ} \mathrm{C}^{-1}$ for $0.4 \mathrm{wt} \%$ DWCNTs/ RTM6 which represents a reduction of $-4.5 \%$. After vitrification we determined: $C_{p}=1.71 \mathrm{~J} \mathrm{~g}^{-1}{ }^{\circ} \mathrm{C}^{-1}$ for the RTM6 resin and $C_{p}=1.65 \mathrm{~J} \mathrm{~g}^{-1}{ }^{\circ} \mathrm{C}^{-1}$ for the DWCNTs/RTM6 mixture which represent a reduction of $-3.5 \%$. The decrease of $C_{p}$ can be related to an increase of the thermal conductivity of the matrix by the addition of DWCNTs which in turn leads to the acceleration the onset of the reaction. Abdalla et al. ${ }^{17}$ in their Modulated DSC results on neat epoxy resin and epoxy resin filled with 1 wt $\%$ of fluorinated multi walled CNTs, they reported similar changes on

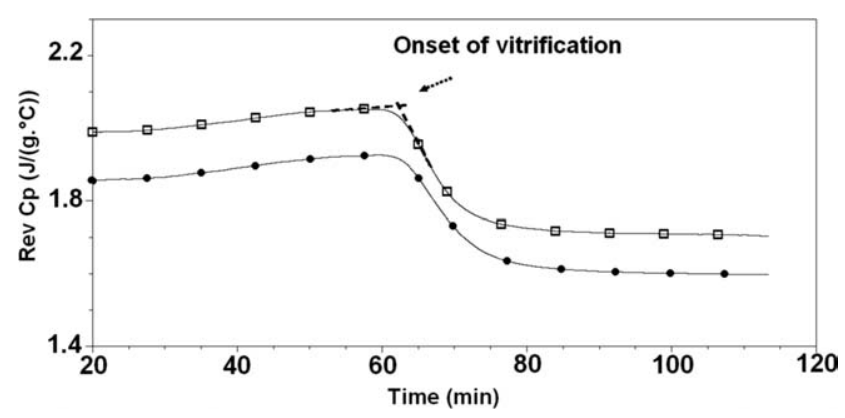

Figure 4 Reversible heat capacity $\left(\operatorname{Rev} C_{p}\right.$ ) from quasiisothermal scan at $160^{\circ} \mathrm{C}$ for the neat RTM6 epoxy resin $(\square)$ and 0.4 wt \% DWCNTs filled RTM6 epoxy resin $(\bullet)$.

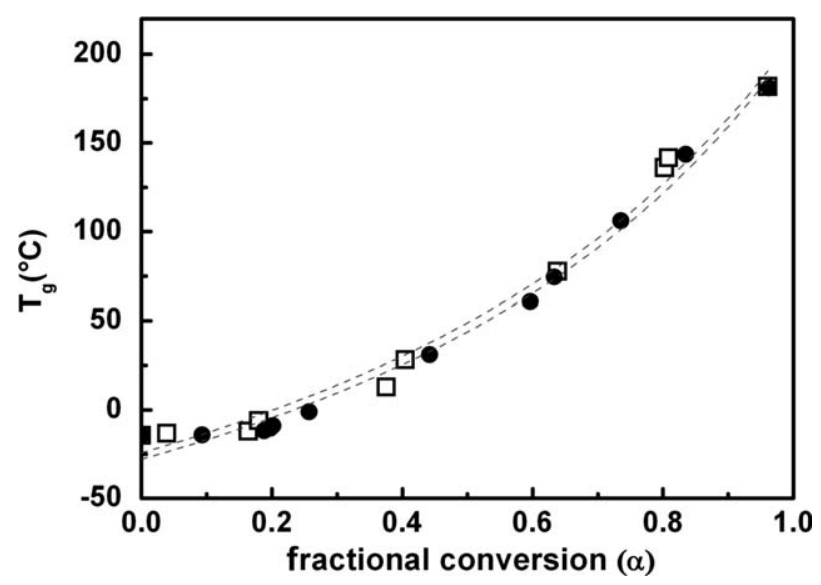

Figure 5 Glass transition $T_{g}$ dependence on the fractional degree of conversions for neat RTM6 epoxy resin ( $\square$ ), 0.4 wt \% DWCNTs filled RTM6 epoxy resin mixture (0) and the dashed lines represent the fit with Eq. (9).

the $C_{p}$, that is, the decrease of $C_{p}$ when CNTs are added. This diminution of $C_{p}$ is related to the reduction of the molecular mobility induced by the presence CNTs in the epoxy network.

The glass transition temperature $T_{g}$ is closely linked to the thermomechanical stability of epoxy resins. We study in this section the effect of DWCNTs on the evolution of $T_{g}$. The increase of the $T_{g}$ with respect to the fractional conversion is shown in the Figure 5. The experimental data were fitted using the Pascault and Williams ${ }^{46}$ equation Eq. (9):

$$
\frac{T_{g}(\alpha)-T g_{0}}{T_{g_{\infty}}-T g_{0}}=\frac{\lambda \alpha}{1-[(1-\lambda) \alpha]}
$$

where $\lambda=\left(\Delta C_{p} \infty / \Delta C_{p} 0\right)$, and $\Delta C_{p} \infty, \Delta C_{p}$ o are the specific heat increment at $T_{g}$ of the fully cured epoxy

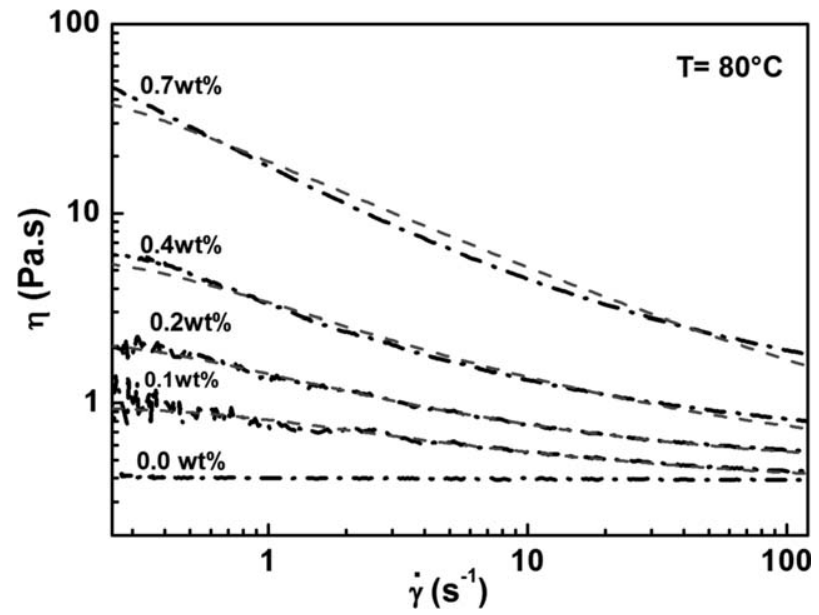

Figure 6 Shear flow viscosity at $80^{\circ} \mathrm{C}$ for various concentrations of DWCNTs; experimental data (-.-.) and CarreauYasuda model (---). 


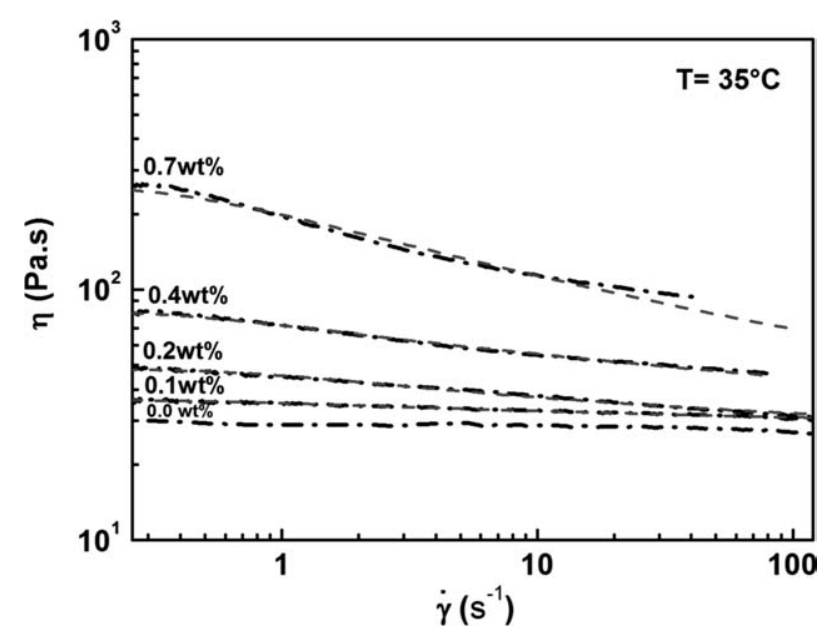

Figure 7 Shear flow viscosity at $35^{\circ} \mathrm{C}$ for various concentrations of DWCNTs; experimental data (-.-.) and CarreauYasuda model (---).

network and the uncured epoxy monomers, respectively. Here $\lambda$ was treated as an adjustable parameter and was determined to be $0.44 \pm 0.05$.

Using Eq. (9) to fit the evolution of $T_{g}$ with respect to temperature we determine $T_{g} 0$ and $T_{g} \infty_{0} T_{g} 0$ is found to be $-27^{\circ} \mathrm{C} \pm 4^{\circ} \mathrm{C}$ and $T_{g} \infty$ is $209^{\circ} \mathrm{C} \pm 8^{\circ} \mathrm{C}$ which is a good agreement with the results determined previously by fitting Eq. (5) to the experimental results shown in Figure 3.

The evolution of the glass transition temperature with respect to the fractional conversion for the RTM6 epoxy resin filled with 0.4 wt \% DWCNTs is the same as for the neat RTM6 epoxy resin. Although this percentage of filler is enough to make an electrical percolation network, the high degree of crosslinks of the epoxy resin seems to restrain the effect of the CNTs on the glass transition temperature. This is unlike the lower degree of crosslinked

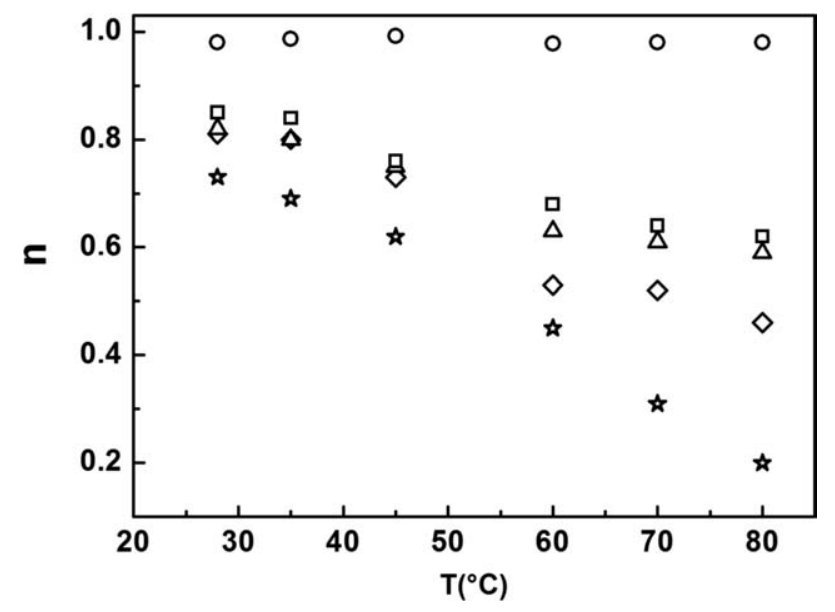

Figure 8 Shear thinning index-exponent $n$ versus temperature for different DWCNTs concentration: Neat RTM6 resin $(\mathrm{O}), 0.1 \mathrm{wt} \%$ DWCNTs $(\square), 0.2$ wt \% DWCNTs $(\nabla)$, 0.4 wt \% DWCNTs $(\diamond), 0.7$ wt \% DWCNTs (ઐ).

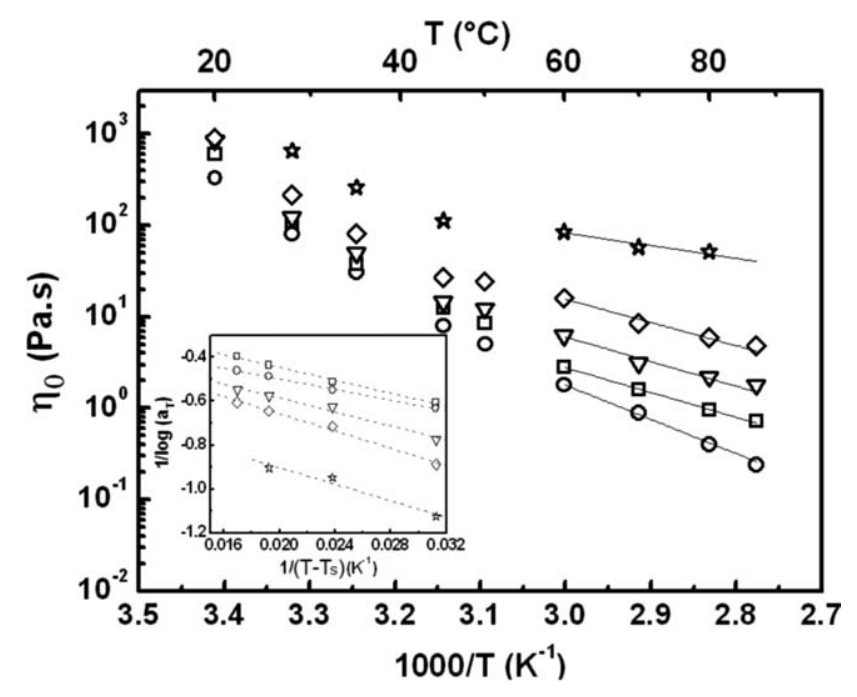

Figure 9 Shear flow viscosity $\eta_{0}(1 / T)$ with respect to (1/ T) for different DWCNTs weight concentration: Neat RTM6 resin (O), 0.1 wt \% DWCNTs $(\square), 0.2$ wt \% DWCNTs $(\nabla), 0.4$ wt \% DWCNTs $(\diamond), 0.7$ wt \% DWCNTs (ㄴ), Andrade-Eyring model [Eq. (7); -] and WLF model [Eq. $(8) ; \cdots]$.

epoxy matrices, ${ }^{47,48}$ rubbery matrices, ${ }^{49}$ and some thermoplastics $^{50}$ for which a comparable amount of carbon nanotubes leads to an increase of the glass transition temperature. For further investigation of the effect of CNTs on the glass transition of the epoxy resin we are currently studying the main mechanical relaxation as function of DWCNTs contents.

Influence of DWCNTs on the shear flow properties of the RTM6 epoxy resin

In this part the shear viscosity property of the liquid RTM6 epoxy resin is studied at different isothermal temperature $\left(28,35,45,50,60,70\right.$, and $\left.80^{\circ} \mathrm{C}\right)$ and for different concentration of DWCNTs. The temperature does not exceed $80^{\circ} \mathrm{C}$ thus the reaction rate of the RTM6 epoxy resin is neglected and $\frac{d a}{d t} \approx 0 \Rightarrow$ the shear viscosity can be expressed as function of the following parameters: $\eta=\eta(T, \dot{\gamma}, \phi)$, where $\phi$ is the DWCNTs volume content. Here, we present separately the influence of the temperature and the DWCNTs content on the shear viscosity.

The RTM6 epoxy resin has a Newtonian behavior in the temperature range studied. The addition of CNTs to this Newtonian suspending medium increases significantly the shear viscosity at low shear rate and as the shear rate increases, the shear viscosity decreased asymptotically, see Figures 6 and 7 . If we keep increasing the shear rate we expect that the viscosity of the CNTs/epoxy reaches the suspending medium viscosity. This is a typical shear-thinning behavior and is commonly observed in many suspensions and polymer composites. ${ }^{51}$ We introduced earlier the Carreau-Yasuda model 
TABLE II

Thermal Activation Energy for Viscous Flow and the Pre-exponential Factor [Eq. (4)]

\begin{tabular}{lcc}
\hline Wt \% DWCNTs & $E_{a}\left(\mathrm{~kJ} \mathrm{~mol}^{-1}\right)$ & $A(\mathrm{~Pa} \mathrm{~s})$ \\
\hline Neat resin & 72 & $1.0 \times 10^{-11}$ \\
0.1 & 52 & $2.1 \times 10^{-8}$ \\
0.2 & 51 & $5.6 \times 10^{-8}$ \\
0.4 & 49 & $4.8 \times 10^{-7}$ \\
0.7 & 27 & $5.0 \times 10^{-3}$ \\
\hline
\end{tabular}

[Eq. (6)] which describes in our case the shear-thinning effect of the DWCNTs on the RTM6 epoxy resin. For all concentrations of DWCNTs and all temperatures studied the shear viscosity data were fitted to the Eq. (6). In the following paragraphs we analyse the parameters of this equation.

The shear thinning index (exponent $\mathrm{n}$ ) determined from the fitting is plotted versus temperature for various concentrations of DWCNTs see Figure 8, $n$ decreases with increasing the temperature and DWCNTs content. We find that the shear-thinning effect is more pronounced at high temperatures and when DWCNTs content is increased.

The shear thinning behavior was experimentally studied by Ma et al. ${ }^{52}$ using in situ optical observations during shear experiments; they concluded that an aggregation and a progressive orientation of the aggregate of CNTs occur in the suspended medium (epoxy resin). Our experimental data of shear flow viscosity are very similar to the authors ${ }^{52}$ and Battisti et al. ${ }^{53}$

When we examine the zero-shear viscosity $\left(\eta_{0}\right)$ of all RTM6/DWCNTs mix and we show its dependency with respect to temperature, see Figure 9, we can distinguish two domains:

For $T>60^{\circ} \mathrm{C}$, the plot of $\log \left(\eta_{0}\right)$ with respect to the inverse of temperature (Fig. 9) shows a linear dependency which reveals thermally activated processes. In this temperature domain, $\log \left(\eta_{0}\right)$ versus $1 / T$ is fitted using the Andrade-Eyring model Eq. (7). The parameters are listed in Table II. The activation energy for viscous flow decreases with increasing DWCNTs content, indicating the decreasing of the sensitivity to change of temperature when CNTs are added to the neat epoxy resin.

Whereas at $T<60^{\circ} \mathrm{C}$ the WLF model [Eq. (8)] shows a good agreement with our experimental
TABLE III

Parameters of the WLF Model [Eq. (8)]

\begin{tabular}{lcllc}
\hline Wt \% DWCNTs & $C_{2}^{S}(\mathrm{~K})$ & $C_{1}^{S}$ & $f_{s} / \mathrm{B}$ & $\alpha_{f}\left(\mathrm{~K}^{-1}\right)$ \\
\hline Neat resin & 98 & 6.66 & 0.065 & $6.63 \times 10^{-4}$ \\
$0.1 \%$ & 67.86 & 4.6 & 0.094 & $1.38 \times 10^{-3}$ \\
$0.2 \%$ & 56.43 & 3.57 & 0.122 & $2.16 \times 10^{-3}$ \\
$0.4 \%$ & 46.73 & 3.84 & 0.113 & $2.41 \times 10^{-3}$ \\
$0.7 \%$ & 43.61 & 1.92 & 0.226 & $5.18 \times 10^{-3}$ \\
\hline
\end{tabular}

data. This is due to the glass transition temperature of the studied RTM6 epoxy resin $\left(T_{g}=-14.5^{\circ} \mathrm{C}\right)$. On the basis of these results, we can state that at temperature lower than $60^{\circ} \mathrm{C}$ the macromolecular mobility of the RTM6 epoxy resin and all DWCNTs/ RTM6 content are affected by the cooperative mobility of the polymer, hence the zero shear viscosity shows a WLF dependency. The plot of $1 / \log \left(a_{T}\right)$ versus $1 /\left(T-T_{s}\right)$ presents a linear relationship for $T<$ $60^{\circ} \mathrm{C}$ see Figure 9. The experimental data are fitted to WLF, Eq. (8). From the slope and the intercept we determine the constants $C_{1}^{S}$ and $C_{2}{ }^{S}$ for each concentration of DWCNTs. $f_{s}$ and $\alpha_{f}$ at $T_{s}$ are reported in Table III. These results show that the addition of DWCNTs increases monotonically the free volume fraction $\left(f_{s}\right.$ increases) of the low molecular weight epoxy-amine monomers. Accordingly, this means that DWCNTs generate free volume in the RTM6 epoxy-amine monomers system.

At infinite shear rate we expect that the $\eta_{\infty}$ for all DWCNTs/RTM6 epoxy mixtures to be the same as the viscosity of the neat RTM6 resin. The $\eta_{\infty}$ plotted versus temperature is the same as the plot (O) shown in the Figure 9.

The parameter $\tau$ (s) of the Eq. (6) shows a temperature and DWCNTs concentration dependence. This parameter is determined by curve fitting and values of $\tau$ are presented in Table IV. It is seen that the value of $\tau$ decreases with increasing temperature and increases with increasing DWCNTs wt $\%$. We can also note that the temperature dependence is much stronger for the higher DWCNTs wt \%. The parameter $\tau$ can be associated to the relaxation time of the polymer thus its elasticity. ${ }^{54}$ The more we add CNTs to the epoxy resin the higher elastic property the polymer exhibit, whereas when the temperature is increased the polymer shows a shorter relaxation time.

TABLE IV

Parameter $\tau$ (s) from Eq. (6) Determined by the Fit of the Shear Viscosity Curves for Each Concentration of DWCNTs and Isothermal Temperature

\begin{tabular}{|c|c|c|c|c|c|c|}
\hline Wt \% DWCNTs & $\tau(\mathrm{s})$ at $28^{\circ} \mathrm{C}$ & $\tau(\mathrm{s})$ at $35^{\circ} \mathrm{C}$ & $\tau(\mathrm{s})$ at $45^{\circ} \mathrm{C}$ & $\tau(\mathrm{s})$ at $60^{\circ} \mathrm{C}$ & $\tau(\mathrm{s})$ at $70^{\circ} \mathrm{C}$ & $\tau(\mathrm{s})$ at $80^{\circ} \mathrm{C}$ \\
\hline Neat resin & - & - & - & - & - & - \\
\hline 0.1 & 2.92 & 2.06 & 1.8 & 1.66 & 1.6 & 1.5 \\
\hline 0.2 & 2.94 & 2.42 & 1.84 & 1.7 & 1.63 & 1.55 \\
\hline 0.4 & 4.49 & 3.4 & 2.81 & 2.25 & 2 & 1.89 \\
\hline 0.7 & 7.6 & 5.11 & 4.47 & 4.13 & 3.82 & 3.5 \\
\hline
\end{tabular}




\section{CONCLUSIONS}

In this study Double Walled CNTs filled epoxy resin where studied from monomer part till the formation of infinite epoxy network by DSC. The DWCNTs are chemically inert at the cure conditions thus reaction rates of the RTM6 epoxy resin and the DWCNTs filled epoxy resin present the same shape. A shift of reaction the rate toward shorter time is observed for the DWCNTs-filled epoxy resin, we assigned this modification to the change in the heat capacity $\left(C_{p}\right)$ of the material.

Shear flow experiments were performed at a temperature $T \leq 80^{\circ} \mathrm{C}$ to avoid initiation of the epoxyamine reaction. The shear viscosity shows two temperature dependence: For $60^{\circ} \mathrm{C} \leq T \leq 80^{\circ} \mathrm{C}$ the viscosity of the epoxy resin and the DWCNTs/ epoxy resin mix exhibit an Arrhenian dependence with respect to temperature and the activation energy decreases with the increase of the DWCNTs wt $\%$. Whereas for a temperature domain $T<60^{\circ} \mathrm{C}$ the complex relaxation processes near the glass transition temperature $T_{g}$ o induces a shear viscosity of the epoxy resin and the DWCNTs/ epoxy resin mix controlled by the free volume. The fractional free volume $\left(f_{s}\right)$ at the chosen reference temperature $\left(T_{s}\right.$ $=28^{\circ} \mathrm{C}$ ) increases with respect to DWCNTs content.

\section{References}

1. Veedu, V. P.; Cao, A.; Li, X.; MA, K.; Soldano, C.; Kar, S.; Ajayan, P. M.; Ghasemi-Nejhad, M. N. Nat Mater 2006, 5, 457.

2. Kim, J. A.; Seong, D. G.; Kang, T. J.; Youn, J. R. Carbon 2006, 44, 1898.

3. Qiu, J.; Zhang, C.; Wang, B.; Liang, R.Nanotechnology 2007, 18,275708 .

4. Moniruzzaman, M.; Winey, K. I. Macromolecules 2006, 39, 5194.

5. Zhu, J.; Kim, J.; Peng, H.; Margrave, J. L.; Khabashesku, V. N.; Barrera, E. V. Nano Lett 2003, 3, 1107.

6. Wang, S.; Liang, Z.; Liu, T.; Wang, B.; Zhang, C. Nanotechnology 2006, 17, 1551.

7. Song, Y. S.; Youn, J. R. Carbon 2005, 43, 1378.

8. Park, H.; Zhao, J.; Lu, J. P. Nano Lett 2006, 6, 916.

9. Špitalský, Z.; Krontiras, C. A.; Georga, S. N.; Galiotis, C. Composites Part A 2009, 40, 778.

10. Barrau, S.; Demont, P.; Perez, E.; Peigney, A.; Laurent, Ch.; Lacabanne, C. Macromolecules 2003, 36, 9678.

11. Zhou, T.; Wanga, X.; Wang, T. Polym Int 2009, 58, 445.

12. Xie, H.; Liu, B.; Yuan, Z.; Shen, J.; Cheng, R. J Polym Sci Part B: Polym Phys 2004, 42, 3701.

13. Puglia, D.; Valentini, L.; Armentano, I.; Kenny, J. M. Diam Relat Mater 2003, 12, 827.

14. Tao, K.; Yang, S.; Grunlan, J. C.; Kim, Y.; Dang, B.; Deng, Y.; Thomas, R. L.; Wilson, B. L.; Wei, X. J Appl Polym Sci 2006, $102,5248$.

15. Deng, H.; Cao, Q.; Wanga, X.; Chen, Q.; Kuang, H.; Wang, X. Mat Sci Eng A-Struct 2011, 528, 5759.

16. Qiu, J.; Wang, S. Mater Chem Phys 2010, 121, 295.

17. Abdalla, M.; Dean, D.; Robinson, P.; Nyairo, E. Polymer 2008, 49, 3310.
18. Dutta, A.; Ryan, M. E. J Appl Polym Sci 1979, 24, 635.

19. Dijkstra, D. J.; Cirstea, M.; Nakamura, N. Rheol Acta 2010, 49, 769.

20. Advani, S. G.; Fan, Z. Proceedings of the 8th International Conference on Numerical Methods in Industrial Forming Processes. AIP Conf. Proc. 2004, 712, 1619.

21. Kim, K. S.; Rhee, K. Y.; Lee, K. H.; Byun, J. H.; Park, S. J. J Ind Eng Chem 2010, 16, 572.

22. Hough, L. A.; Islam, M. F.; Janmey, P. A.; Yodh, A. G. Phys Rev Lett 2004, 93, 168102-4.

23. Hobbie, E. K. Rheol Acta 2010, 49, 323.

24. Kovacs, J. Z.; Mandjarov, R.; Blisnjuk, T.; Prehn, K.; Sussiek, M.; Müller, J.; Schulte, K.; Bauhöfer, W. Nanotechnology 2009, 20, 155703.

25. Bauhöfer, W.; Schulz, S. C.; Eken, A. E.; Skipa, T.; Lellinger, D.; Alig, I.; Tozzi, E. J.; Klingenberg, D. J. Polymer 2010, 51, 5024.

26. Eken, A. E.; Tozzi, E. J.; Klingenberg, D. J.; Bauhöfer, W. J Appl Phys 2011, 109, 084342.

27. Aduriz, X. A.; Lupi, C.; Boyard, N.; Bailleul, J. L.; Leduc, D.; Sobotka, V.; Lefevre, N.; Chapeleau, X.; Boisrobert, C.; Delaunay, D. Compos Sci Technol 2007, 67, 3196.

28. Kazilas, M. C.; Partridge, I. K. Polymer 2005, 46, 5868.

29. Karkanas, P.; Partridge, I. K.; Attwood, D. Polym Int 1996, 41, 183.

30. Terekhina, S.; Fouvry, S.; Salvia, M.; Bulanovb, I. Wear 2010, $269,632$.

31. Flahaut, E.; Bacsa, R.; Peigney, A.; Laurent, Ch. Chem Commun 2003, 21, 1442.

32. Flahaut, E.; Laurent, Ch.; Peigney, A. Carbon 2005, 4, 375.

33. Sourour, S.; Kamal, M. R. Thermochim Acta 1976, 14, 41.

34. Torre, L.; Lelli, G.; Kenny, J. M. J Appl Polym Sci 2004, 94, 1676.

35. Lee, S. N.; Chiu, M. T.; Lin, H. S. Polym Eng Sci 1992, 32, 1037.

36. Terenzi, A.; Vedova, C.; Lelli, G.; Mijovic, J.; Torrea, L.; Valentini, L.; Kenny, J. M. Compos Sci Technol 2008, 68, 1862.

37. Gonzalez-Romero, V. M.; Cassilas, N. Polym Eng Sci 1989, 29, 295.

38. Nielsen, L. E. J Macromol Sci-Rev Macromol Chem 1969, C3, 69.

39. Carreau, P. J. J Rheol 1972, 16, 99.

40. Yasuda, K.; Armstrong, R. C.; Cohen, R. E. Rheol Acta 1981, 20, 163.

41. Eyring, H. J Chem Phys 1936, 4, 283.

42. Williams, M. L.; Landel, R. F.; Ferry, J. D. J Am Chem Soc 1955, 77, 3701.

43. Vogel, D.H. Phys Z 1921, 22, 645.

44. Fulcher, G. S. J Am Ceram Soc 1925, 8, 339.

45. Tammann, G.; Hesse, W. Z Anorg Allg Chem 1926, 156, 245.

46. Pascault, J. P.; Williams, R. J. J. J Polym Sci Part B: Polym Phys 1990, 28, 85.

47. Gojny, F. H.; Schulte, K. Compos Sci Technol 2004, 64, 2303.

48. Suave, J.; Coelho, L. A. F.; Amico, S. C.; Pezzin, S. H. Mat Sci Eng A-Struct 2009, 509, 57.

49. Sui, G.; Zhong, W. H.; Yang, X. P.; Yu, Y. H.; Zhao, S. H. Polym Adv Technol 2008, 19, 1543.

50. Gong, X.; Liu, J.; Baskaran, S.; Voise, R. D.; Young, J. S. Chem Mater 2000, 12, 1049.

51. Gupta, R. K. Polymer and Composite Rheology, 2nd ed.; Marcel Dekker Inc.: New York, 2000; Chapter 10, pp 224-264.

52. Ma, A. W. K.; Chinesta, F.; Mackley, M. R.; Ammar, A. Int J Mater Form 2008, 1, 83.

53. Battisti, A.; Skordos, A. A.; Partridge, I. K. Compos Sci Technol 2009, 69, 1516.

54. Han, C. D. Rheology and Processing of Polymer Materials; Polymer Rheology; Oxford University Press: Oxford, 2007; Vol 1, Chapter 6, pp 203-246. 